\title{
Competition dependence of retrieval-induced forgetting in motor memory
}

\author{
Tobias Tempel $^{2}$ - Alp Aslan ${ }^{1}$ - Christian Frings ${ }^{2}$
}

Published online: 14 December 2015

(C) Psychonomic Society, Inc. 2015

\begin{abstract}
In two experiments, we examined the competition dependence of retrieval-induced forgetting (RIF) in motor memory. Participants learned sequential finger movements as responses to letter stimuli. The learning phase comprised two parts. In both parts, half of the motor sequences were to be executed at one of two locations (the left or right side of a keyboard) by pressing the corresponding response keys. Retrieval practice of half of the motor sequences at one location induced forgetting of the nonpracticed motor sequences at that location. However, RIF was prevented in Experiment 1 when retrieval practice took place before the nonpracticed items had even been encoded. In Experiment 2, RIF was prevented by intentionally forgetting the nonpracticed motor sequences prior to retrieval practice. These results suggest that precluding competition by related items during retrieval practice precluded them from being affected by RIF. The present findings support an inhibitory account and speak against the alternative assumptions that associative blocking or a mental context change causes RIF.
\end{abstract}

Keywords Motor memory - Body movement .

Retrieval-induced forgetting $\cdot$ Inhibition

Retrieval practice of previously studied information is typically beneficial for accessing the retrieved information later on (e.g.,

Tobias Tempel

tempel@uni-trier.de

1 Psychology Department, Martin Luther University of Halle-Wittenberg, 06099 Halle, Germany

2 Fachbereich I-Psychologie, University of Trier, 54286 Trier, Germany
Karpicke \& Roediger, 2008). Recent studies have shown that such beneficial effects of retrieval also occur in motor action. For example, Boutin, Panzer, \& Blandin (2013) demonstrated that retrieval practice improved the generalization of a dynamic arm movement skill (see also Boutin, Panzer, Salesse, \& Blandin, 2012). Kromann, Jensen, and Ringsted (2009) showed that retrieval practice at the end of a resuscitation course for medical students enhanced the retention of resuscitation skills. In addition to beneficial effects, however, retrieval practice also can impair access to nonretrieved information when retrieval is selective - only pertaining to a subset of information.

Typically, retrieval-induced forgetting (RIF) is investigated in the retrieval practice paradigm (Anderson, Bjork, \& Bjork, 1994), which consists of three main phases. In the learning phase, participants study several sets of items in combination with a shared (category) cue that defines the specific set of items. In the subsequent retrieval-practice phase, participants are cued to recall half of the studied items from half of the sets. In the test phase, recall performance for all items is tested. The recall of practiced items $(\mathrm{Rp}+\mathrm{items})$, nonpracticed items from the practiced sets ( $\mathrm{Rp}-$ items), and nonpracticed items from nonpracticed sets (Nrp items) is then compared. $\mathrm{Rp}+$ items usually profit from retrieval practice and are recalled better than Nrp items. RIF manifests itself in significantly lower recall of $\mathrm{Rp}$ - than of Nrp items. Whereas most studies on RIF have used verbal items, Tempel and Frings (2013) recently demonstrated that RIF affects motor sequences. Participants first learned 12 sequential finger movements (SFM), each consisting of two consecutive keypresses in response to a letter stimulus. Six items involved two fingers on the left hand, and six involved two fingers on the right hand. After retrieval practice of three items related to one hand, a final recall test assessed memory for all items. The results revealed that retrieval practice improved recall of the practiced SFMs but impaired recall of the nonpracticed SFMs on the same hand, 
as compared to the baseline SFMs on the other hand, thus extending the finding of RIF to motor memory.

RIF has mostly been explained in terms of an inhibitory mechanism serving to resolve interference during selective retrieval (Anderson, 2003; Anderson et al., 1994; Anderson \& Spellman, 1995; Wimber, Alink, Charest, Kriegeskorte, \& Anderson, 2015). The inhibitory account of RIF assumes that during (selective) retrieval practice of the $\mathrm{Rp}+$ items, the notto-be-retrieved $\mathrm{Rp}$ - items interfere in a competition for conscious recollection. To resolve this interference, the $\mathrm{Rp}+$ items are strengthened while, simultaneously, the $\mathrm{Rp}-$ items are inhibited. The inhibitory account posits that inhibition lowers the activation of the individual memory representations of $\mathrm{Rp}-$ items. Therefore, RIF should also emerge in memory tests that do not provide information present at learning as retrieval cues (cue independence). The inhibitory account further posits that it is necessary to attempt to retrieve $\mathrm{Rp}+$ items during retrieval practice for RIF to occur (retrieval specificity) and that the strength of activation of $\mathrm{Rp}+$ items during retrieval practice does not influence the amount of resulting RIF (strength independence). Finally, it posits that the occurrence of inhibition depends on the strength of competition during retrieval practice (competition dependence). $\mathrm{Rp}$ - items are inhibited only if they actually do compete with the to-beretrieved $\mathrm{Rp}+$ items. Although the inhibitory account of RIF is well-supported by many studies (for a recent meta-analysis, see Murayama, Miyatsu, Buchli, \& Storm, 2014), the basic finding of RIF can also be explained by noninhibitory mechanisms. For instance, an alternative account assumes that $\mathrm{Rp}+$ items block the $\mathrm{Rp}$ - items; that is, recall of $\mathrm{Rp}$ - items suffers from stronger interference in the test phase than does recall of Nrp items (Raaijmakers \& Jakab, 2013). According to this noninhibitory account, the occurrence of RIF does not depend on competition during retrieval practice.

First evidence on competition dependence was provided by Anderson et al. (1994) who found that only items with strong (semantic) item-cue associations were affected by RIF, but not items with weak item-cue associations (for similar results, see Bäuml, 1998). This may suggest that $\mathrm{Rp}-$ items were not inhibited because their associations to the shared category cue were too weak to trigger competition with $\mathrm{Rp}+$ items. However, results regarding the effects of strength of the semantic item-cue associations of Rp- items on RIF have been mixed (Williams \& Zacks, 2001) and other manipulations of item strength have failed to provide evidence in favor of competition dependence (Jakab \& Raaijmakers, 2009). Moreover, RIF has been found to also occur for previously unknown words - that is, for items lacking semantic representations (Tempel \& Frings, 2015c) - as well as for verbal materials organized into ad-hoc-defined categories - for example, personality traits linked to an arbitrary name (Dunn \& Spellman, 2003; Macrae \& MacLeod, 1999). Finally, RIF also occurs for newly learned perceptual stimuli (Ciranni \&
Shimamura, 1999) and motor actions (Reppa, Worth, Greville, \& Saunders, 2013; Tempel \& Frings, 2013; Tempel, Loran, \& Frings, 2015) which suggests that weak or purely episodic item-cue associations can suffice for RIF to occur.

However, there are further approaches to testing competition dependence. Storm, Bjork, and Bjork (2007) combined the list method of directed forgetting with the retrievalpractice paradigm. During the learning phase, participants were instructed to forget some items after having encoded them, whereas other items were to be retained for the later test. RIF did not occur for to-be-forgotten items, but only for to-be-retained items (see also Aslan \& Bäuml, 2014; Bäuml \& Samenieh, 2010, 2012). This finding suggests that lowered accessibility due to intentional forgetting prevented competition. Chan, Erdman, and Davis (2015) recently showed that RIF affects $\mathrm{Rp}$ - items only if retrieval practice takes place after the $\mathrm{Rp}$ - items have been encoded. Instead of including retrieval practice after termination of the learning phase, several study blocks and retrieval-practice blocks alternated. A nocompetition group, for whom the retrieval of $\mathrm{Rp}+$ items was required before the $\mathrm{Rp}$ - items had been encoded, did not show RIF, which occurred only in a competition group, who retrieved $\mathrm{Rp}+$ items in later blocks, after $\mathrm{Rp}-$ items had been encoded as well. In the present investigation, we used both modifications of the retrieval-practice paradigm to analyze inhibition in motor memory. We examined whether RIF of motor actions also depends on competition during retrieval practice.

The properties of phenomena in verbal memory cannot be readily transferred to motor memory. Since different brain systems are likely involved in encoding and processing verbal versus motor content (e.g., Krakauer \& Shadmehr, 2006), processes of interference and inhibition may operate differently, as well. In general, current memory research has unnecessarily neglected motor action. A notable exception has been work by Anderson (2003) who explicitly linked inhibition in memory with behavioral inhibition in motor action, and found that both achieved a kind of response override (see also Anderson \& Weaver, 2009). Yet, empirical investigations of retrieval dynamics in motor memory are scarce. Hence, studies are needed that will examine whether motor memory operates similarly or differently from verbal memory.

RIF of motor actions has been found to be retrieval-specific. In a study by Tempel and Frings (2015b), replacing retrieval practice with extra study did not induce forgetting of related motor sequences, although it did enhance memory for the extra-studied motor sequences in the same way that retrieval practice strengthens $\mathrm{Rp}+$ sequences. The retrieval specificity of RIF in motor memory was also demonstrated in a study by Tempel et al. (2015) in which participants learned dance figures (i.e., complex whole-body movements). Only selective retrieval practice of subsets of the dance figures caused RIF for related dance figures, whereas selective restudy did not impair later recall of the related dance figures. Although these findings have 
demonstrated the retrieval specificity of RIF for motor actions occurring with one type of processing (retrieval practice) but not with other types (extra study), they do not speak to the issue of whether RIF is competition-dependent. Indeed, to come up with a strong(er) test of competition dependence, it would be necessary to compare conditions that differed only in the amounts of competition, but not in the type of processing. This was exactly what we did in the present experiments.

\section{Experiment 1}

In Experiment 1, we adopted the approach of Chan et al. (2015). The learning phase consisted of two blocks. In both learning blocks, participants learned six SFMs, each consisting of the consecutive movement of three fingers of the right hand. The SFMs were organized into two categories by location. Half of the SFMs were executed by pressing marked keys on the left side of a standard keyboard, and the other half by pressing marked keys on the right side of the keyboard. The location of movement execution, thus, served as a category to organize encoding of the SFMs. It has been shown that different features can organize the storage of motor sequences in such a way as to entail retrieval dynamics that produce RIF. Those features comprise effectors (e.g., Tempel \& Frings, 2014b), movement directions (Tempel \& Frings, 2015a) and location (Reppa et al., 2013). Here, retrieval practice pertained to three SFMs on either the left or the right side of the keyboard. Two groups were compared: Whereas the competition group performed retrieval practice after Learning Block 2 (L2), the no-competition group performed retrieval practice after Learning Block 1 (L1). Finally, memory for all 12 SFMs was assessed in the test phase. We expected RIF to occur in the competition but not in the no-competition group.

\section{Method}

Participants A total of 64 undergraduate students at the University of Trier participated in the experiment. They either received course credit for their participation or were paid six euros.

Design The study had a 2 (Retrieval Practice Group: competition, no competition) $\times 4$ (Item Type: Rp+, Rp-, Nrp-, Nrp+) mixed design with repeated measures on the last factor. $\mathrm{Rp}-$ items were tested before $\mathrm{Rp}+$ items, in order to preclude any output interference by the Rp+ items (cf. Anderson et al., 1994; Roediger \& Schmidt, 1980). Rp- items, accordingly, were compared to the first three $\mathrm{Nrp}$ items tested ( $\mathrm{Nrp}-$ ), whereas $\mathrm{Rp}+$ items were compared to the last three Nrp items tested $(\mathrm{Nrp}+)$.

Material The experiment was conducted using Dell Optiplex 755 PCs with Eizo FlexScan S1901 monitors and standard
German QWERTZ keyboards. The software PXLab (Irtel, 2007) served for running the experiment. The items consisted of 12 three-finger movements to be performed with the fingers of the right hand. Six were performed by pressing keys on the left side of the keyboard, six by pressing keys on the right side of the keyboard. The letters $a$ to $f$ were the stimuli for movements on the left side, and the letters $u$ to $z$ for movements on the right side (see the Appendix). During the learning phase a letter appeared on the corresponding side of the screen, together with an animation of the three-finger movement (see Fig. 1, upper section). The participants placed their right index finger, middle finger, ring finger, and pinkie on either the $Y, X, C$, and $V$ keys of the main keyboard or the 1, 2, 3, and Enter keys within the numeric keypad on the ride side of the keyboard. These response keys were marked with labels hiding the letter names. Below the letter, a display of the right hand demonstrated which fingers should be moved by showing three consecutively flashing fingers (the first finger was colored yellow, the second finger blue, and the third finger yellow again; $200 \mathrm{~ms}$ per flash). After the display of the hand disappeared, participants could perform the movement. If the performed sequence was incorrect, a feedback appeared, displaying "Fehler!" (English: "Error!").

Procedure The experiment consisted of five phases (L1, L2, retrieval practice, distractor task, and final recall). Instructions were given on the screen and summarized by an experimenter. On learning trials, participants had to consecutively press three keys in response to a displayed letter accompanied by an animation graphic display showing which digits should be moved. In L1 as well as in L2, each item was presented ten times. On retrieval-practice trials, participants had to retrieve an SFM in response to its letter stimulus. A letter appeared on the screen, and the participant entered the corresponding item while his or her fingers again rested on the marked keys on the corresponding side of the keyboard (see Fig. 1, lower section). No feedback about the accuracy of the performed three-finger movements was given, as is usual in most studies on RIF.

Depending on the experimental group, the retrievalpractice phase took place either after L2 (competition) or after L1 (no competition). Both groups started by learning three SFMs on the left and three SFMs on the right side of the keyboard in L1. In the no-competition group, retrieval practice of the three SFMs on one side from L1 then followed. After retrieval practice, another three SFMs on the left and another three SFMs on the right side were learned in L2. Thus, for this group L1 always comprised $\mathrm{Rp}+$ and $\mathrm{Nrp}+$ items, whereas L2 comprised $\mathrm{Rp}-$ and Nrp - items. In contrast, L2 followed immediately after L1 in the competition group. After L2, retrieval practice took place of three SFMs that had been learned in either L1 or L2.

In the competition group, we counterbalanced whether the $\mathrm{Rp}+$ or $\mathrm{Rp}-$ items were presented in L1 or L2, as well as 


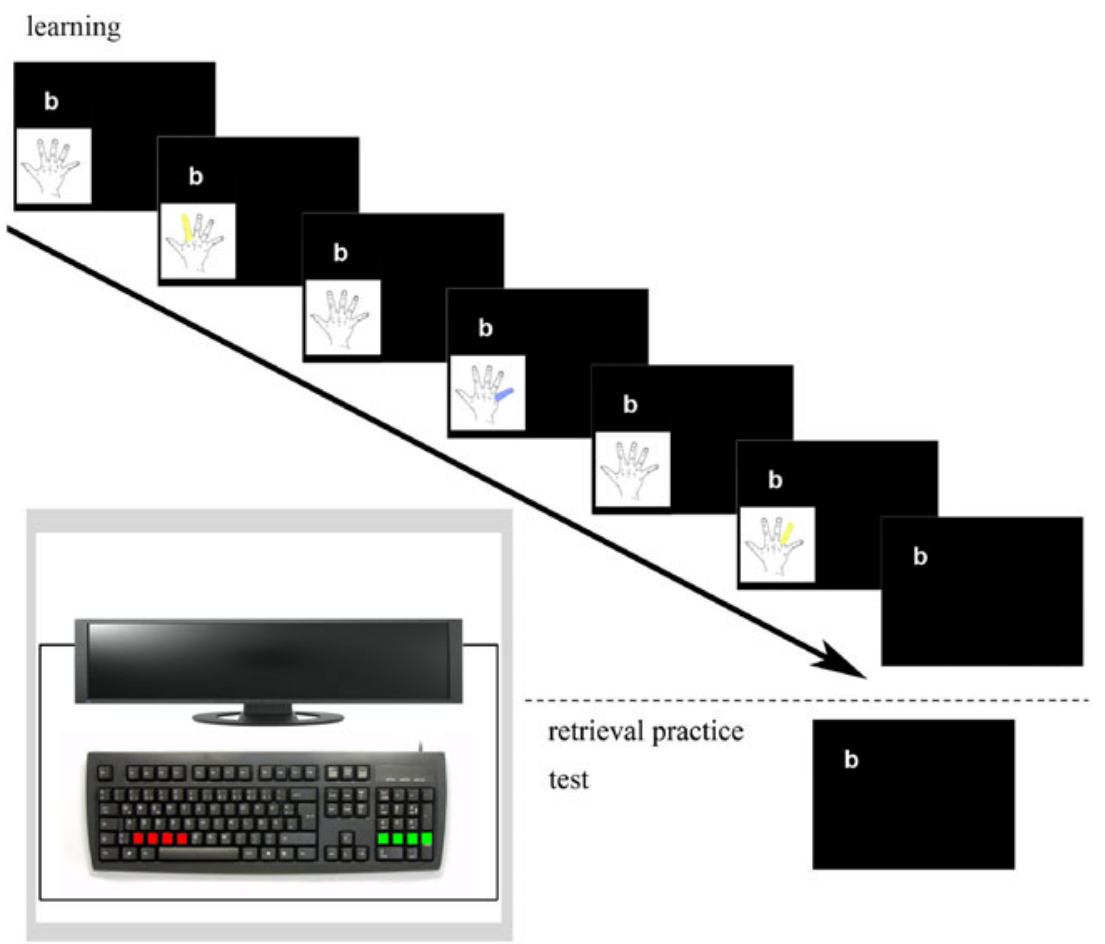

Fig. 1 The upper section depicts a trial in the learning phase. It starts with display of the letter stimulus, together with a drawing of the right hand. After $400 \mathrm{~ms}$, one finger illuminates yellow for $200 \mathrm{~ms}$, then a second finger illuminates blue for $200 \mathrm{~ms}$, and then a third finger illuminates yellow for $200 \mathrm{~ms}$. The illuminations are separated by intervals of

whether Nrp- or Nrp+ items were presented in L1 or L2. The three items of one type ( $a b c, d e f, u v w$, or $x y z$ ) were always presented within the same part of the learning phase. These counterbalancing strategies precluded a confounding of learning block with item type in the competition group. Restricting the encoding of one item type to either L1 or L2 would have limited any results to this specific assignment, since the learning block could potentially moderate the impact of retrieval practice. Furthermore, we counterbalanced the assignments of SFMs to L1 and L2 in both conditions.

After the 3-min distractor task (a Sudoku puzzle), the test phase presented participants with 12 letter stimuli in the same fashion as during retrieval practice. The items of one keyboard side were tested in succession; that is, the test was blocked by keyboard side. We counterbalanced whether the test sequence started with the retrieval-practiced or the non-retrievalpracticed keyboard side.

\section{Results}

Preliminary analyses revealed that counterbalancing did not affect the overall pattern of results, all $p s>.216$. We ran a 2 (Group: competition, no competition) $\times 2$ (Item Type: Rp-, Nrp-) analysis of variance (ANOVA) to examine RIF; the results for all experiments are reported in Table 1 . The main
$200 \mathrm{~ms}$ in which the uncolored hand is shown. Subsequently, the hand display disappears and the participant can enter the sequential finger movement (SFM) just illustrated. The lower section depicts a trial in either the retrieval-practice or the test phase. A stimulus is given, and the participant is supposed to perform the corresponding SFM.

effect of group was significant, $F(1,62)=12.29, p=.001, \eta_{\mathrm{p}}{ }^{2}$ $=.17$, as was the main effect of item type, $F(1,62)=6.50, p=$ $.013, \eta_{\mathrm{p}}{ }^{2}=.10$. The interaction was also significant, $F(1,62)=$ $4.05, p=.049, \eta_{\mathrm{p}}{ }^{2}=.06$. Simple-effects analyses showed that significantly fewer $\mathrm{Rp}$ - than $\mathrm{Nrp}$ - items were recalled in the competition group, $F(1,62)=10.40, p=.002, \eta_{\mathrm{p}}{ }^{2}=.14$, but that the numbers of recalled $\mathrm{Rp}-$ and $\mathrm{Nrp}$ - items did not differ significantly in the no-competition group, $F<1$ (see Fig. 2).

Table 1 Mean percentages of items recalled as a function of item type and experimental group

\begin{tabular}{lllll}
\hline & \multicolumn{3}{l}{ Item Type } & \\
\cline { 2 - 5 } & $\mathrm{Rp}-$ & $\mathrm{Nrp}-$ & $\mathrm{Nrp}+$ & $\mathrm{Rp}+$ \\
\hline Experiment 1 & & & & \\
Competition & 38 & 55 & 51 & 59 \\
No competition & 73 & 75 & 54 & 59 \\
Experiment 2 & & & & \\
Remember L1 & 39 & 53 & 44 & 68 \\
Forget L1 & 40 & 35 & 56 & 81 \\
\hline
\end{tabular}

$\mathrm{Rp}+=$ retrieval-practiced items; $\mathrm{Rp}-=$ nonpracticed items on the retrieval-practiced keyboard side; $\mathrm{Nrp}-=$ retrieval-induced forgetting baseline; $\mathrm{Nrp}+=$ retrieval benefit baseline; $\mathrm{L} 1=$ Learning Block 1 


\section{Experiment 1}
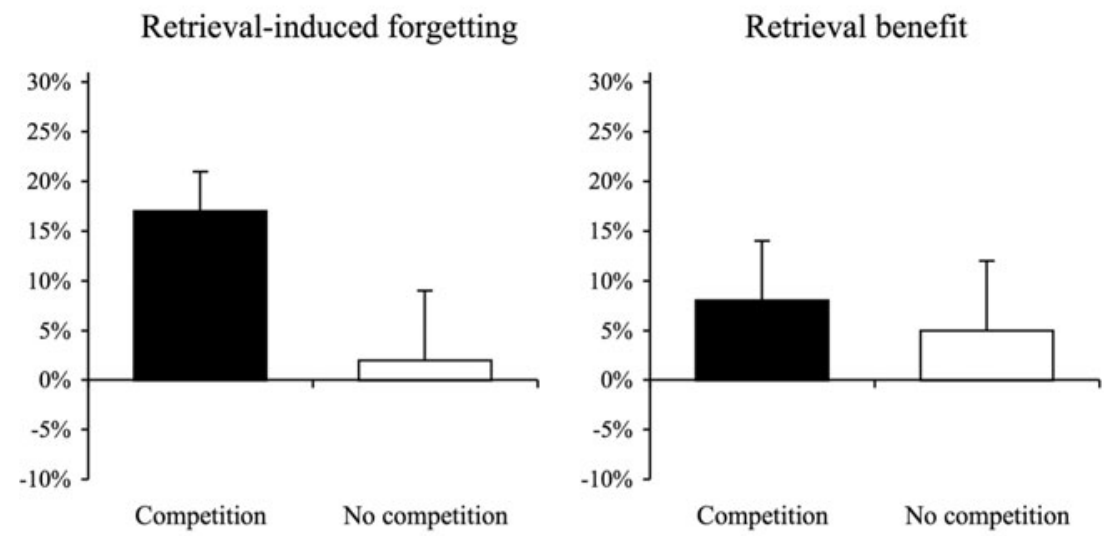

\section{Experiment 2}

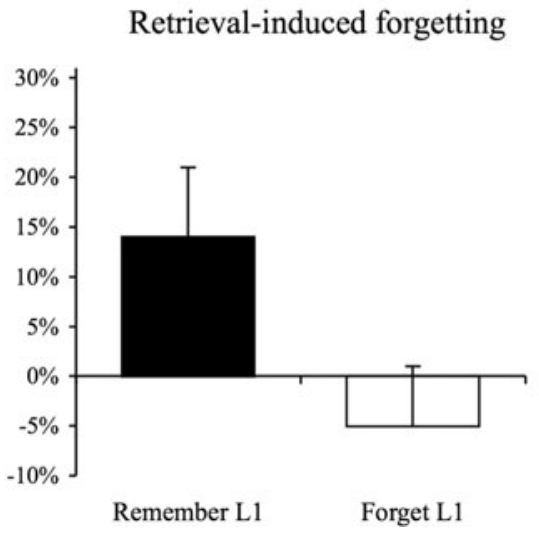

Fig. 2 Effects of retrieval-induced forgetting (percentage of recalled Nrp- items - percentage of recalled Rp-items) and the retrieval benefit (percentage of recalled $\mathrm{Rp}+$ items - percentage of recalled Nrp+ items),

In a 2 (Group: competition, no competition) $\times 2$ (Item Type: $\mathrm{Rp}+, \mathrm{Nrp}+)$ ANOVA, we examined the Rp+ retrieval benefit. Although slightly more $\mathrm{Rp}+$ than Nrp+ items were recalled, the main effect of item type did not reach significance, $F(1,62)=2.27, p=.137$. The main effect of group was also not significant, nor was the interaction, $F<1$.

\section{Discussion}

The results matched the recent findings by Chan et al. (2015). A standard RIF effect occurred when retrieval practice took place after all items had been encoded. However, no RIF occurred in the no-competition group, who retrieved $\mathrm{Rp}+$ items before the Rp-items had been encoded. These results suggest that the RIF was a consequence of competition during retrieval practice. Such competition could not arise, of course, with regard to $\mathrm{Rp}$ - items that had not been encoded before, because they did not yet exist in the memories of participants. Associative blocking cannot account for the observed RIF effect, because at test all items had been encoded, and all items were
Retrieval benefit

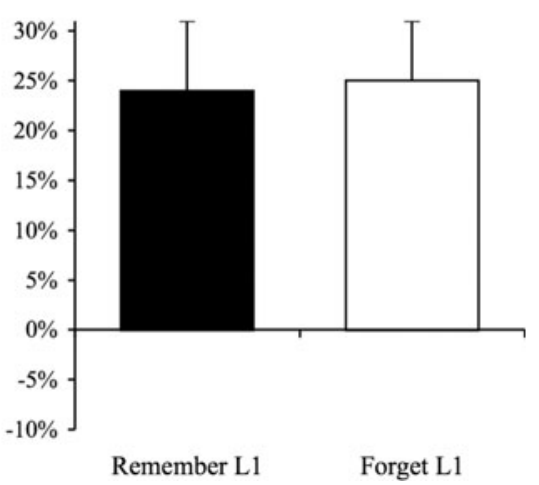

as a function of experimental group. Error bars depict standard errors of the means

associated with their respective category. Moreover, Rp+ items were not recalled significantly more than Nrp+ items, although there was a tendency in this direction. This weak retrieval benefit further suggests that strengthening of the $\mathrm{Rp}+$ items was not what caused RIF. Indeed, we found the $\mathrm{Rp}+$ retrieval benefit effects for motor sequences to be less robust and of smaller size than the RIF effects from previous experiments that controlled for the effects of output interference on Rp- items (Tempel \& Frings, 2013, 2014a, 2014b) because the later recall of $\mathrm{Rp}+$ items subjects them to output interference by the $\mathrm{Rp}$ - items, diminishing the retrieval benefit effects. Still, the lack of a significant $R p+$ retrieval benefit is rather untypical, as compared to the majority of RIF studies. Perhaps the present findings depended on the weakness of the $\mathrm{Rp}+$ retrieval benefit, although this seems unlikely, because the effects were equally weak in both groups, whereas there was a significant difference with regard to RIF.

An additional difference between the groups was in their recall levels of Rp- and Nrp-items. Obviously, recall of these items in the no-competition group benefited from the items' 
more recent presentation in L2 and prior retrieval practice (Pastötter, Schicker, Niedernhuber, \& Bäuml, 2011). In contrast, $\mathrm{Rp}-$ and $\mathrm{Nrp}$ - items had been presented equally in L1 or L2 in the competition group. Different recall levels due to the effect of on-average more recent learning cannot explain why the recall of $\mathrm{Rp}$ - items was not impaired relative to Nrpitems. Still, we modulated competition without this potential caveat in Experiment 2.

\section{Experiment 2}

The goal of Experiment 2 was to conceptually replicate the results of Experiment 1 using a different way to manipulate competition during retrieval practice. To this end, we followed previous work and employed the directed-forgetting task, to lower the accessibility of Rp- items after their encoding (Storm et al., 2007). The learning phase again consisted of two parts, and two groups were compared. In both groups, retrieval practice now followed L2. However, the groups differed from each other regarding an instruction after L1. Participants either were simply told that the first part of the learning phase would now be followed by its second part (remember-L1 group) or were told to forget the so-far-presented motor sequences (forget-L1 group). $\mathrm{Rp}-$ and $\mathrm{Nrp}$ - items were presented in $\mathrm{L} 1$, and $\mathrm{Rp}+$ and Nrp+ items in L2. A further change pertained to the retrieval practice trials. In order to increase the strengthening of $\mathrm{Rp}+$ items, we included feedback.

We assumed that the forget instruction would eliminate competition by $\mathrm{Rp}$ - items during retrieval practice, because it would markedly segregate L1 from L2. According to the current literature on directed forgetting, a "forget" cue should lower the accessibility of L1 due to the inhibition of L1 (Pastötter \& Bäuml, 2010) or to a change in internal context (Sahakyan \& Kelley, 2002). This lowered accessibility should preclude competition from L1 items on the recall of L2 items. Therefore, we expected RIF to occur in the remember-L1 but not in the forget-L1 group.

\section{Method}

Participants A total of 64 undergraduate students at the University of Trier participated in the experiment. They either received course credit for their participation or were paid six euros.

Design The study had a 2 (Group: forget L1, remember L1) $\times$ 4 (Item Type: $\mathrm{Rp}+, \mathrm{Rp}-, \mathrm{Nrp}-, \mathrm{Nrp}+$ ) mixed design with repeated measures on the last factor.

Material and procedure We used the same materials as in Experiment 1. The procedure also was identical, with the following exceptions. Retrieval practice took place after L2 in both groups. After L1, the participants in the forget-L1 group were instructed to forget the so-far-presented items. They were told that the previous learning trials had only served as a warm-up. The importance of trying to forget the previously presented motor sequences and only to retain the subsequently to-be-learned motor sequences was emphasized. In the remember-L1 group, the participants were informed that they had finished the first part of the learning phase and were going to continue with the second part. In both groups, a 3-min break separated L1 and L2 (Tempel \& Frings, 2015). Participants were instructed to relax and to concentrate for the beginning of the real experiment (forget-L1 group) or, respectively, for the continuation of the learning phase (remember-L1 group). Also, participants were provided with immediate feedback in the retrieval-practice trials. After participants had executed an SFM in response to a given letter, the same animation graphic display as during the learning trials appeared on the screen, depicting the correct SFM. Rp-and Nrp-items were presented in L1, and Rp+ and Nrp+ items were presented in L2 in both groups.

\section{Results}

In a 2 (Group) $\times 2$ (Item Type: Rp-, Nrp-) ANOVA, we examined RIF. The main effect of group was not significant, $F(1,62)=1.03, p=.313$, nor was the main effect of item type, $F(1,62)=1.48, p=.228$. However, the interaction was significant, $F(1,62)=4.80, p=$ $.032, \eta_{\mathrm{p}}{ }^{2}=.07$. Simple-effects analyses showed that significantly fewer $\mathrm{Rp}$ - than Nrp- items were recalled in the remember-L1 group, $F(1,62)=5.81, p=.019$, $\eta_{\mathrm{p}}{ }^{2}=.09$, but the numbers of recalled $\mathrm{Rp}-$ and $\mathrm{Nrp}-$ items did not differ significantly in the forget-L1 group, $F<1$.

A 2 (Group) $\times 2$ (Item Type: $\mathrm{Rp}+$, Nrp+) ANOVA was run to examine $\mathrm{Rp}+$ retrieval benefits. The main effect of item type was significant, $F(1,62)=30.37, p<.001, \eta_{\mathrm{p}}{ }^{2}=.33$ : More $\mathrm{Rp}+$ than $\mathrm{Nrp}+$ items were recalled. The main effect of group was not significant, $F(1,62)=2.62, p=.110$, nor was the interaction, $F<1$.

A further analysis compared recall of Nrp- items in the remember-L1 and forget-L1 groups. Significantly fewer Nrp- items were recalled in the forget-L1 group, $t(62)=$ $1.92, p=.029$, one-tailed, indicating success at intentionally forgetting $\mathrm{L} 1$ items.

\section{Discussion}

Directed forgetting eliminated RIF, suggesting that the intentional forgetting of $\mathrm{Rp}$ - items prevented them from triggering competition during retrieval practice. The impaired recall of $\mathrm{Nrp}-$ in the forget-L1 as compared to the remember-L1 group represents a cost effect of directed forgetting and shows that participants 
successfully segregated L1 and L2, although it does not specify whether inhibition or a change in internal context accounted for the reduced accessibility in the test phase. The inclusion of feedback during retrieval practice entailed a strong retrieval benefit for $\mathrm{Rp}+$ items. Despite this strengthening, $\mathrm{Rp}$ - items were not impaired as a consequence of retrieval practice, but their accessibility remained on the same level as Nrp- items in the forget-L1 group.

\section{General discussion}

Using SFMs, we examined in two experiments whether RIF in motor memory is competition-dependent. In both experiments, we found that selective retrieval of a subset of previously encoded SFMs could induce forgetting of other previously encoded SFMs, thus replicating prior work by showing that RIF generalizes to motor memory (e.g., Tempel \& Frings, 2013). More importantly, and going beyond the prior work, we additionally found RIF to be eliminated when retrieval practice took place before nonpracticed SFMs had even been encoded (Exp. 1), and when nonpracticed SFMs had been encoded but, after encoding, had been cued to be forgotten (Exp. 2). Two different manipulations of competition strength, thus, demonstrated that RIF is eliminated when items that possess the potential to interfere during retrieval, due to associations to a common retrieval cue with tobe-retrieved items, are prevented from interfering when their activation during retrieval practice is precluded. In Experiment 1, such activation could not occur because the potentially interfering items had not yet been encoded. In Experiment 2, intentional forgetting eliminated their activation.

In addition to conceptually replicating the effect of timing retrieval practice before versus after encoding $\mathrm{Rp}$ - items, we can rule out that peculiarities of Experiment 1 beyond that manipulation might have accounted for the moderation of RIF. First, the recall levels of Rp-and Nrp-items were higher than the recall levels of $\mathrm{Rp}+$ and $\mathrm{Nrp}+$ items in the nocompetition group, because the former items benefited from their on-average more recent encoding in L2 as well as the prior retrieval practice, but Experiment 2 controlled for equal encoding positions of all item types in the remember-L1 and forget-L1 groups. Second, the lack of a retrieval benefit effect in Experiment 1 led us to provide feedback in the retrievalpractice trials in Experiment 2. As expected, feedback increased the retrieval benefit effect considerably but did not affect RIF. This also corresponds to the postulate of strength independence in the inhibitory account. RIF occurred independently of the degree of strengthening of Rp+ items in the groups with competition. Beside a 3-min break between L1 and L2, feedback was the only difference between these two groups, both showing RIF.

We adapted a paradigm used by Chan et al. (2015) with verbal learning materials to the use of motor sequences. In contrast to to-be-learned words, the motor sequences in the present study were newly acquired; that is, they did not possess any preexisting representations in memory. Thus, any activation of other than the retrieval-practiced motor sequences of the same category during retrieval practice was precluded with certainty in the no-competition group. However, because intentional forgetting of $\mathrm{Rp}-$ items also prevented RIF, the nonexistence of items in memory was not necessary to prevent competition, but the retrograde exclusion of a set of already learned motor sequences from becoming activated during retrieval practice sufficed. Using novel motor actions as the tobe-learned materials permitted us to demonstrate the functional equivalence of segregating L1 and L2 by means of directed forgetting to a complete lack of representation of L2 in memory.

The observed competition dependence suggests that a noninhibitory account assuming that associative blocking causes RIF (Raaijmakers \& Jakab, 2013) cannot explain the results of the present study. Another noninhibitory account assumes that RIF is the consequence of a mental context change during retrieval practice (Jonker, Seli, \& MacLeod, 2013). The Rp+ items, therefore, are associated with two internal contexts: the encoding context and the retrieval-practice context. In the test phase, the retrieval-practice context is reactivated for $\mathrm{Rp}+$ as well as $\mathrm{Rp}-$ items, but the encoding context is reactivated in test trials for Nrp items. The activation of the retrieval-practice context disadvantages the accessibility of $\mathrm{Rp}$ - as compared to Nrp items, because $\mathrm{Rp}$ - items had not been associated with that context, but only with the encoding context. Thus, RIF reflects the activation of the "wrong" context when Rp- items are to be recalled in the test phase. In particular, the results of Experiment 2 may require that additional assumptions be incorporated if the results are to be explained by the mentalcontext-change account. The forget-L1 instruction influenced $\mathrm{Rp}-$ as well as Nrp- items, whereas a mental context change during retrieval practice should only have affected Rp- items. Given equal initial states of activation, the disadvantageous activation of the retrieval-practice context only for $\mathrm{Rp}-$ items, but not for Nrp- items, still should have impaired the recall of $\mathrm{Rp}-$ items. The present challenge of the context-change account of RIF is in line with very recent work indicating that a change in mental context is neither necessary nor sufficient for RIF to occur (Buchli, Storm, \& Bjork, 2015; Soares, Polack, \& Miller, 2015).

Retrieval dynamics in motor memory resemble those in declarative memory. After first evidence that selective 
retrieval of motor sequences that are represented in memory in an organized manner can impair memory for related motor sequences (Reppa et al., 2013; Tempel \& Frings, 2013). it has been shown that such effects are indeed retrievalspecific; that is, retrieval-free types of practice do not hamper the related motor sequences (Tempel \& Frings, 2015b; Tempel et al., 2015). The present findings suggest that RIF in motor memory occurs only when there is (sufficient) competition between the to-be-retrieved and related motor actions. The demonstrated competition dependence, together with our evidence on retrieval specificity and strength independence, suggests that an inhibitory mechanism serves to resolve such interference between body movements. Thus, the present findings suggest domain-general inhibition in memory (MacLeod, 2007). Moreover, motor inhibition is also involved in the voluntary control of action execution-investigated, for example, in go/no-go or stop-signal tasks (Logan \& Cowan, 1984). Neuroimaging studies have shown that the inhibitory control mechanisms involved in voluntarily stopping actions and inhibiting memories overlap (Anderson \& Hanslmayr, 2014). Consistently, Schilling, Storm, and Anderson (2014) reported a positive relationship between motor inhibition and memory inhibition, with individuals who showed better performance in the stop-signal task also showing higher amounts of RIF in verbal memory. RIF of motor sequences may provide a further way to scrutinize whether inhibitory control works in a supramodal way or whether the distinct inhibitory mechanisms involved in stopping action can be separated from the processes that resolve interference in motor memory. For example, electroencephalography could be used in such investigations to measure interference, cognitive inhibition, and motor inhibition, because each of these concepts has been linked to distinct oscillatory patterns in previous studies (e.g., Sauseng, Gerloff, \& Hummel, 2013; Staudigl, Hanslmayr, \& Bäuml, 2010).

Practical implications of the present study may pertain to training motor sequences - for example, in sports or music exercises. RIF can be an unwelcome side effect of practice. In fact, a multitude of studies on RIF have proven its robustness over a wide variety of modalities. In motor memory, RIF has been shown for finger movements (Tempel \& Frings, 2013), hand movements (Tempel \& Frings, 2014b, 2015a), as well as complex whole-body movements (Tempel et al., 2015). Nevertheless, there are ways to prevent RIF, such as the intentional forgetting of potentially competing memories. The effects of directed forgetting typically are not highly persistent (Abel \& Bäuml, 2013). Therefore, the focused intention to forget a set of body movements may be used to prevent RIF without running the risk of weakening the to-be-forgotten movements in the long run.
Author note This research was supported by Grant Number TE 891/31 from the German Research Council (Deutsche Forschungsgemeinschaft).

\section{Appendix: Items}

\begin{tabular}{llll}
\hline First Finger & Second Finger & Third Finger & Stimulus \\
middle finger & index finger & pinkie & $\mathrm{a}$ \\
index finger & pinkie & ring finger & $\mathrm{b}$ \\
ring finger & middle finger & index finger & $\mathrm{c}$ \\
pinkie & index finger & middle finger & $\mathrm{d}$ \\
middle finger & ring finger & index finger & $\mathrm{e}$ \\
index finger & middle finger & pinkie & $\mathrm{f}$ \\
pinkie & middle finger & ring finger & $\mathrm{u}$ \\
ring finger & pinkie & middle finger & $\mathrm{V}$ \\
index finger & ring finger & middle finger & $\mathrm{W}$ \\
pinkie & ring finger & index finger & $\mathrm{x}$ \\
ring finger & index finger & pinkie & $\mathrm{y}$ \\
middle finger & pinkie & ring finger & $\mathrm{z}$ \\
\hline
\end{tabular}

\section{References}

Abel, M., \& Bäuml, K.-H. T. (2013). Sleep can eliminate list-method directed forgetting. Journal of Experimental Psychology: Learning, Memory, and Cognition, 39, 946-952. doi:10.1037/ a0030529

Anderson, M. C. (2003). Rethinking interference theory: Executive control and the mechanisms of forgetting. Journal of Memory and Language, 49, 415-445. doi:10.1016/j.jml.2003.08.006

Anderson, M. C., Bjork, R. A., \& Bjork, E. L. (1994). Remembering can cause forgetting: Retrieval dynamics in long-term memory. Journal of Experimental Psychology: Learning, Memory, and Cognition, 20, 1063-1087. doi:10. 1037/0278-7393.20.5.1063

Anderson, M. C., \& Hanslmayr, S. (2014). Neural mechanisms of motivated forgetting. Trends in Cognitive Sciences, 18, 279-292. doi:10. 1016/j.tics.2014.03.002

Anderson, M. C., \& Spellman, B. A. (1995). On the status of inhibitory mechanisms in cognition: Memory retrieval as a model case. Psychological Review, 102, 68-100. doi:10.1037/0033-295X. 102.1.68

Anderson, M. C., \& Weaver, C. (2009). Inhibitory control over action and memory. In L. R. Squire (Ed.), The encyclopedia of neuroscience (pp. 153-163). Amsterdam: Elsevier.

Aslan, A., \& Bäuml, K.-H. T. (2014). Later maturation of the beneficial than the detrimental effect of selective memory retrieval. Psychological Science, 25, 1025-1030. doi:10.1177/ 0956797613519270

Bäuml, K.-H. (1998). Strong items get suppressed, weak items do not: The role of item strength in output interference. Psychonomic Bulletin \& Review, 5, 459-463. doi:10.3758/BF03208822

Bäuml, K.-H., \& Samenieh, A. (2010). The two faces of memory retrieval. Psychological Science, 21, 793-795. doi:10.1177/ 0956797610370162 
Bäuml, K.-H., \& Samenieh, A. (2012). Selective memory retrieval can impair and improve retrieval of other memories. Journal of Experimental Psychology: Learning, Memory, and Cognition, 38, 488-494. doi:10.1037/a0025683

Boutin, A., Panzer, S., Salesse, R. N., \& Blandin, Y. (2012). Testing promotes effector transfer. Acta Psychologica, 141, 400-407. doi: 10.1016/j.actpsy.2012.09.014

Boutin, A., Panzer, S., \& Blandin, Y. (2013). Retrieval practice in motor learning. Human Movement Science, 32, 1201-1213. doi:10.1016/j. humov.2012.10.002

Buchli, D. R., Storm, B. C., \& Bjork, R. A. (2015). Explaining retrievalinduced forgetting: A change in mental context between the study and restudy practice phases is not sufficient to cause forgetting. Quarterly Journal of Experimental Psychology. doi:10.1080/ 17470218.2015.1076866

Chan, C. K., Erdman, M. R., \& Davis, S. D. (2015). Retrieval induces forgetting, but only when nontested items compete for retrieval: Implication for interference, inhibition, and context reinstatement. Journal of Experimental Psychology: Learning, Memory, and Cognition, 41, 1298-1315. doi:10.1037/0278-7393.20.5.1063

Ciranni, M. A., \& Shimamura, A. P. (1999). Retrieval-induced forgetting in episodic memory. Journal of Experimental Psychology: Learning, Memory, and Cognition, 25, 1403-1414. doi:10.1037/ 0278-7393.25.6.1403

Dunn, E. W., \& Spellman, B. A. (2003). Forgetting by remembering: Stereotype inhibition through rehearsal of alternative aspects of identity. Journal of Experimental Social Psychology, 39, 420-433. doi:10.1016/S0022-1031(03)00032-5

Irtel, H. (2007). PXLab: The Psychological Experiments Laboratory (Version 2.1.11) [Online]. Mannheim, Germany: University of Mannheim. Available from www.pxlab.de

Jakab, E., \& Raaijmakers, J. G. W. (2009). Retrieval-induced forgetting without competition: Testing the retrieval specificity assumption of the inhibition theory. Journal of Experimental Psychology: Learning, Memory, and Cognition, 35, 607-617. doi:10.1037/ a0015264

Jonker, T. R., Seli, P., \& MacLeod, C. M. (2013). Putting retrievalinduced forgetting in context: An inhibition-free, context-based account. Psychological Review, 120, 852-872. doi:10.1037/a0034246

Karpicke, J. D., \& Roediger, H. L., III. (2008). The critical importance of retrieval for learning. Science, 319, 966-968. doi:10.1126/science. 1152408

Krakauer, J. W., \& Shadmehr, R. (2006). Consolidation of motor memory. Trends in Neurosciences, 29, 58-64. doi:10.1016/j. tins.2005.10.003

Kromann, C. B., Jensen, M. L., \& Ringsted, C. (2009). The effect of testing on skills learning. Medical Education, 43, 21-27. doi:10. $1111 / j .1365-2923.2008 .03245 . x$

Logan, G. D., \& Cowan, W. B. (1984). On the ability to inhibit thought and action: A theory of an act of control. Psychological Review, 91, 295-327. doi:10.1037/0033-295X.91.3.295

MacLeod, C. M. (2007). The concept of inhibition in cognition. In D. S. Gorfein \& C. M. MacLeod (Eds.), Inhibition in cognition (pp. $3-$ 23). Washington: American Psychological Association.

Macrae, C. N., \& MacLeod, M. D. (1999). On recollections lost: When practice makes imperfect. Journal of Personality and Social Psychology, 77, 463-473. doi:10.1037/0022-3514.77.3.463

Murayama, K., Miyatsu, T., Buchli, D., \& Storm, B. C. (2014). Forgetting as a consequence of retrieval: A meta-analytic review of retrievalinduced forgetting. Psychological Bulletin, 140, 1383-1409. doi:10. 1037/a0037505

Pastötter, B., \& Bäuml, K.-H. T. (2010). Amount of postcue encoding predicts amount of directed forgetting. Journal of Experimental Psychology: Learning, Memory, and Cognition, 36, 54-65. doi:10. 1037/a0017406
Pastötter, B., Schicker, S., Niedernhuber, J., \& Bäuml, K.-H. T. (2011). Retrieval during learning facilitates subsequent memory encoding. Journal of Experimental Psychology: Learning, Memory, \& Cognition, 37, 287-297. doi:10.1037/a0021801

Raaijmakers, J. G. W., \& Jakab, E. (2013). Rethinking inhibition theory: On the problematic status of the inhibition theory for forgetting. Journal of Memory and Language, 68, 98-122. doi:10.1016/j.jml. 2012.10.002

Reppa, I., Worth, E. R., Greville, W. J., \& Saunders, J. (2013). The representation of response effector and response location in episodic memory for newly acquired actions: Evidence from retrievalinduced forgetting. Acta Psychologica, 143, 210-217. doi:10. 1016/j.actpsy.2013.03.007

Roediger, H. L., III, \& Schmidt, S. R. (1980). Output interference in the recall of categorized and paired associate lists. Journal of Experimental Psychology: Human Learning and Memory, 6, 91105. doi:10.1037/0278-7393.6.1.91

Sahakyan, L., \& Kelley, C. M. (2002). A contextual change account of the directed forgetting effect. Journal of Experimental Psychology: Learning, Memory, and Cognition, 28, 1064-1072. doi:10.1037 0278-7393.28.6.1064

Sauseng, P., Gerloff, C., \& Hummel, F. C. (2013). Two brakes are better than one: The neural bases of inhibitory control of motor traces. NeuroImage, 65, 52-58. doi:10.1016/j.neuroimage.2012.09.048

Schilling, C. J., Storm, B. C., \& Anderson, M. C. (2014). Examining the costs and benefits of inhibition in memory retrieval. Cognition, 133, 358-370. doi:10.1016/j.cognition.2014.07.003

Soares, J. S., Polack, C. W., \& Miller, R. R. (2015). Retrieval-induced versus context-induced forgetting: Does retrieval-induced forgetting depend on context shifts? Journal of Experimental Psychology: Learning, Memory, and Cognition. doi:10.1037/xlm0000171

Staudigl, T., Hanslmayr, S., \& Bäuml, K.-H. T. (2010). Theta oscillations reflect the dynamics of interference in episodic memory retrieval. Journal of Neuroscience, 30, 11356-11362. doi:10.1523/ JNEUROSCI.0637-10.2010

Storm, B. C., Bjork, E. L., \& Bjork, R. A. (2007). When intended remembering leads to unintended forgetting. Quarterly Journal of Experimental Psychology, 60, 909-915. doi:10.1080/ 17470210701288706

Tempel, T., \& Frings, C. (2013). Resolving interference between body movements: Retrieval-induced forgetting of motor sequences. Journal of Experimental Psychology: Learning, Memory, and Cognition, 39, 1152-1161. doi:10.1037/a0030336

Tempel, T., \& Frings, C. (2014a). Forgetting motor programmes: Retrieval dynamics in procedural memory. Memory, 22, 11161125. doi:10.1080/09658211.2013.871293

Tempel, T., \& Frings, C. (2014b). Interference within hands: Retrievalinduced forgetting of left and right hand movements. Acta Psychologica, 148, 1-5. doi:10.1016/j.actpsy.2014.01.003

Tempel, T., \& Frings, C. (2015a). Categorization by movement direction: Retrieval-induced forgetting of motor sequences grouped by motion features. Quarterly Journal of Experimental Psychology, 68, 473486. doi:10.1080/17470218.2014.945098

Tempel, T., \& Frings, C. (2015b). How motor practice shapes memory: Retrieval but not extra study can cause forgetting. Memory. doi:10. 1080/09658211.2015.1059858. Advance online publication.

Tempel, T., \& Frings, C. (2015c). Interference in episodic memory: Retrieval-induced forgetting of unknown words. Psychological Research, 79, 795-800. doi:10.1007/s00426-014-0604-2

Tempel, T., \& Frings, C. (2015). Directed forgetting benefits motor sequence encoding. Memory \& Cognition. doi:10.3758/s13421-0150565-8

Tempel, T., Loran, I., \& Frings, C. (2015). Dancing your moves away: How memory retrieval shapes complex motor action. Journal of Experimental Psychology: Applied, 21, 300-312. doi:10.1037/ xap0000052 
Williams, C. C., \& Zacks, R. T. (2001). Is retrieval-induced forgetting an inhibitory process? American Journal of Psychology, 114, 329-354. doi:10.2307/1423685
Wimber, M., Alink, A., Charest, I., Kriegeskorte, N., \& Anderson, M. C. (2015). Retrieval induces adaptive forgetting of competing memories via cortical pattern suppression. Nature Neuroscience, 18, 582589. doi:10.1038/nn.3973 